\title{
Intestinal Tuberculosis Presenting as Massive Lower Gastrointestinal Bleeding: A Rare Presentation in Child
}

\author{
Aji Mathew ${ }^{*}$, Yusuf Parvez ${ }^{2}$ and Sen Thomas ${ }^{2}$ \\ ${ }^{1}$ Department of Paediatrics, Al Jalila Children Hospital, Dubai, UAE; ${ }^{2}$ Department of Paediatrics, Dubai Hospital, Dubai, UAE
}

\begin{abstract}
Background: Intestinal tuberculosis is a form of extra pulmonary tuberculosis that can involve any part of the gastrointestinal tract. It can present with nonspecific symptoms like chronic abdominal pain, anorexia, fatigue, fever, night sweats, weight loss, diarrhea, constipation, or blood in the stools. Acute presentation including intestinal obstruction and perforation have been reported in the literature; however massive lower gastrointestinal bleeding as one of the presenting symptom is rare. The management includes anti-tubercular drugs along with surgical intervention in acute presentation.

Case characteristic: 11-year-old girl was admitted with complaints of fever and vague abdominal pain for one-week duration.

Observation/intervention: The child was having recent weight loss, hypo albuminemia and strongly positive Manteaux test. Ultrasound abdomen revealed multiple pre and para aortic lymphadenopathy. The Computerised tomography (CT) guided biopsy from lymph node was planned but the child developed massive lower gastrointestinal bleeding which was managed surgically.

Conclusion: The child underwent hemi-colectomy with ileostomy and intestinal tuberculosis was confirmed by biopsy. The patient was discharged home on anti-tubercular drugs with further follow up.

Message: Intestinal tuberculosis should be considered as one of the differential diagnosis in a child presenting with massive lower gastrointestinal bleeding.
\end{abstract}

Keywords: Intestinal tuberculosis; Bleeding; Hemi colectomy

\section{INTRODUCTION}

Tuberculous enteritis is a form of extra pulmonary tuberculosis that can involve any part of the gastrointestinal tract. It accounts for 1 to $3 \%$ of TB worldwide and $6^{\text {th }}$ commonest site of extra pulmonary tuberculosis. It can present with nonspecific symptoms like chronic abdominal pain, anorexia, fatigue, fever, night sweats, weight loss, diarrhea, constipation, or blood in the stool. Only $15-20 \%$ of patients have concomitant active pulmonary tuberculosis [1]. Acute presentation including intestinal obstruction and perforation have been reported in the literature; however massive lower gastrointestinal bleeding as one of the presenting symptoms is rare [2]. Fewer than 20 cases in adults have been reported but none have been reported in children as per the existing literature [3]. We report a case of intestinal tuberculosis presented with vague abdominal symptoms who later on developed torrential lower gastrointestinal bleeding requiring urgent surgical intervention.

\section{CASE REPORT}

11-year-old girl was admitted with complaints of fever and vague abdominal pain for one-week duration. There was associated weight loss of around $5 \mathrm{~kg}$. On examination the child was emaciated with growth parameters below $3^{\text {rd }}$ centile. She had mild pallor with hepatosplenomegaly and vague abdominal tenderness over the right lumbar region and peri-umbilical region. Other systemic examination was normal. Initial investigations showed haemoglobin $9 \mathrm{gram} /$ decilitre $(\mathrm{gm} / \mathrm{dl})$, Serum Albumin-1.5 gm/dl. Liver function tests were mildly

*Correspondence to: Aji Mathew, Specialist Paediatrics, Al Jalila Children Hospital, Dubai, UAE, Tel: +971555933183; E-mail: docaji@gmail.com

Received: June 18, 2018; Accepted: May 21, 2019; Published: May 28, 2019

Citation: Mathew A, Parvez Y, Thomas S (2019) Intestinal Tuberculosis Presenting as Massive Lower Gastrointestinal Bleeding: A Rare Presentation in Child. J Bacteriol Parasitol. 10:357.

Copyright: (C) 2019 Mathew A, et al. This is an open-access article distributed under the terms of the Creative Commons Attribution License, which permits unrestricted use, distribution, and reproduction in any medium, provided the original author and source are credited. 
deranged with mild elevation of total bilirubin and mild derangement of coagulation profile. Manteaux test was strongly positive. Stool for occult blood was positive. Ultra sonogram (USG) abdomen showed mild hepatosplenomegaly with multiple enlarged pre and para aortic nodes. X-ray chest and abdomen were normal. The urine routine was normal and didn't reveal proteinuria.

In view of the clinical and lab findings including strongly positive Mantaux test, intestinal tuberculosis was suspected and was planned for Computerised tomography (CT) abdomen and CT guided lymph node biopsy and colonoscopy. The child started having torrential bleeding per rectum on subsequent day prior to CT abdomen. She was managed initially with multiple blood transfusions and fresh frozen plasma infusions. Colonoscopy was tried but couldn't proceed due to massive bleeding. The bleeding became more profuse and patient developed hypovolemic shock and managed by fluid boluses and blood transfusion. But the child continued to bleed massively per rectum, so was immediately taken for emergency laparotomy.

Laparotomy revealed diseased, bluish gangrenous bowel from ileocecal junction to transverse colon with multiple adhesion and multiple enlarged and matted lymph nodes (Figure 1).

She underwent resection of diseased intestine from ileocecal junction to transverse colon, and ileostomy was performed. Intraoperative lymph node biopsy and liver biopsy was performed. Her bleeding was controlled. Intestinal biopsy and lymph node biopsy revealed multiple caseating granulomas suggestive of tuberculous enteritis. Smear from the diseased intestine was positive for Acid fast bacilli. Gastric aspirate which was collected initially was positive for acid fast bacilli and culture grew Mycobacterium tuberculosis. She was started on antituberculous medications. She improved symptomatically and hence discharged with regular follow up.

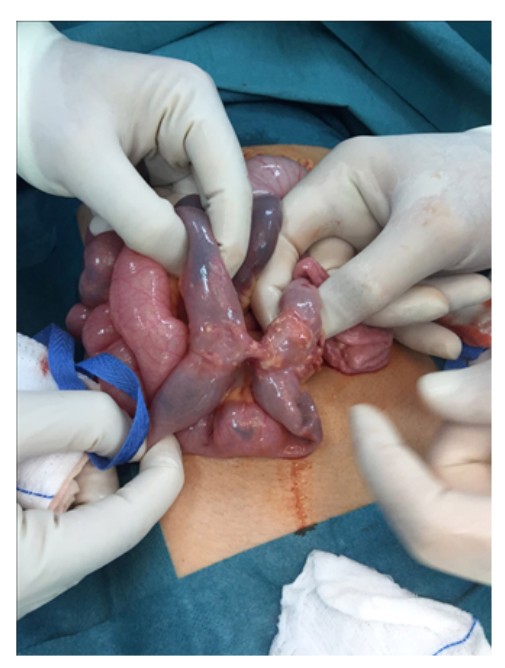

Figure 1: Intestinal tuberculosis suspected in children presenting with massive lower gastrointestinal bleeding.

\section{DISCUSSION}

Abdominal tuberculosis has varied presentation and mimics a variety of diseases like Crohn's disease, Ulcerative colitis and intestinal malignancy. Usually the symptoms are vague with abdominal discomfort, change in bowel habits, weight loss, fever and other constitutional symptoms. The gastrointestinal bleeding is frequently minor and massive bleeding is less common. Singh et al. reported rectal bleeding in $31 \%$ of patients with colonic tuberculosis, and it was massive in $13 \%$ [4]. Overall, tuberculosis accounts for about $4 \%$ of patients with lower gastrointestinal bleeding [5] but massive lower gastrointestinal hemorrhage requiring blood transfusion and surgical intervention is unusual in tuberculous enteritis.

In a study of 130 patients with alimentary tract tuberculosis by Al Karawi et al., the disease was located in the small bowel in 44 patients (33.8\%) and in the large bowel in 29 patients $(22.3 \%)$. Moreover, in their study of 44 patients with small bowel tuberculosis, hemorrhage was observed in only one patient but was observed in three of 29 patients with large bowel tuberculosis [6]. There is a case report of intestinal TB with lower gastrointestinal bleeding managed by endoscopic sclerotherapy but majority needed laparotomy $[7,8]$. Bleeding is most likely due to the ulceration in colon. Ulcers are hyperaemic and can erode into blood vessels and the vessels develop obliterative endarteritis with capillary proliferation leading to massive bleeding [9]. Colonoscopy can aid in locating the bleeding site but in torrential bleeding it is not feasible. $99 \mathrm{mTc}$ red blood cell scintigraphy was useful to locate the bleeding lesion and to make a preoperative diagnosis of hemorrhage from the ileum [10].

There are few case reports of massive intestinal bleeding secondary to intestinal tuberculosis in adults but in children it is extremely rare [11,12]. The hallmark of extra pulmonary TB histopathology is the caseating granuloma [13]. All patients who are diagnosed to have gastrointestinal tuberculosis should undergo treatment with anti-tubercular drugs.

\section{CONCLUSION}

Intestinal tuberculosis should be suspected in children presenting with massive lower gastrointestinal bleeding. Urgent surgical intervention followed by anti-tubercular drugs is the mainstay of treatment.

\section{REFERENCES}

1. Marshall JB. Tuberculosis of the gastrointestinal tract and peritoneum. Am J Gastroenterol. 1993;88(7):989-999.

2. Beppu K, Osada T, Matsumoto K, Shibuya T, Sakamoto N, Kawabe M, et al. Gastrointestinal tuberculosis as a cause of massive bleeding. Med Sci Monit. 2009;15(10):CS151-154.

3. Kela M, Agrawal A, Sharma R, Agarwal R, Agarwal VB. Ileal tuberculosis presenting as a case of massive rectal bleeding. Clin Exp Gastroenterol. 2009;2:129-131.

4. Singh V, Kumar P, Kamal J, Prakash V, Vaiphei K, Singh K, et al. Clinico-colonoscopic profile of colonic tuberculosis. Am J Gastroenterol. 1996;91:565-568.

5. Kapoor VK. Abdominal tuberculosis: the Indian contribution. Indian J Gastroenterol. 1998;17:141-147. 
6. Al Karawi MA, Mohamed AE, Yasawy MI, Graham Dy, Shariq S, Ahmed AM et al. Protean manifestation of gastrointestinal tuberculosis: report on 130 patients. J Clin Gastroenterol. 1995;20(3):225-232.

7. Bhargava DK, Kushwaha AKS, Dasarathy S, Shriniwas, Chopra P. Endoscopic diagnosis of segmental colonic tuberculosis. Gastrointest Endosc. 1992;38:571-574.

8. Park JK, Lee SH, Kim SG, Kim HY, Lee JH, Shim JH, et al. A case of intestinal tuberculosis presenting massive hematochezia controlled by endoscopic coagulation therapy. Korean J Gastroenterol. 2005;45(1):60-63.

9. Sherman HI, Johnson R, Brock T. Massive gastrointestinal bleeding from tuberculosis of small intestine. Am J Gastroenterol. 1978;70(3):314-316.
10. Smith R, Copely DJ, Bolen FH. 99mTc RBC scintigraphy: correlation of gastrointestinal bleeding rates with scintigraphic findings. AJR Am J Roentgenol. 1987;148(5):869-874.

11. Goudarzi HA, Mason LB. Fatal rectal bleeding due to tuberculosis of the cecum. JAMA. 1982;247(5):667-668.

12. Rabkin DG, Caiati JM, Allendorf JA, Treat M. Intractable hematochezia: an unusual presentation of intestinal tuberculosis. Surgery. 2003;133(5):592-593.

13. Tandon HD, Prakash A. Pathology of intestinal tuberculosis and its distinction from Crohn's disease. Gut. 1972;13(4):260-269. 